\title{
Predictive factors of outcome and stroke recurrence in patients with unilateral atherosclerosis-related internal carotid artery occlusion
}

\author{
Wen-Yi Huang, Wei-Chieh Weng, Yu-Yi Chien, Chia-Lun Wu, Tsung-I Peng ${ }^{1}$, Kuan-Hsing Chen ${ }^{2}$ \\ Department of Neurology, Keelung Chang Gung Memorial Hospital, 'Department of Neurology, Keelung Chang Gung Memorial Hospital and \\ Chang Gung University, Taoyuan, Taiwan, ${ }^{2}$ Chang Gung University, Taoyuan, Taiwan
}

\begin{abstract}
Background: Clinical outcome of internal carotid artery (ICA) occlusion is highly variable and the reason is uncertain. Aim: To study the predictive factors of clinical outcome and stroke recurrence in patients with ischemic stroke associated with unilateral atherosclerosis-related ICA occlusion. Settings and Design: Prospective study in neurology department of a single hospital. Materials and Methods: We prospectively studied 66 patients who suffered from first-ever ischemic stroke associated with unilateral atherosclerosis-related ICA occlusion over a period of two years. The end point was death or stroke recurrence. Statistical Analysis: Chi-square or Fisher's exact test was used to analyze predictors of early functional outcome. Multivariate analysis was used to analyze predictors of death or stroke recurrence within two years. Result: Higher age ( $\geq 70$ years) predicted a worse functional outcome $(P=0.049)$. Total anterior circulation syndrome (TACS) was associated with a poor functional outcome $(P<0.001)$, but lacunar syndrome had a better outcome $(P=0.001)$. Stroke in evolution predicted a poor outcome $(P=0.001)$, while those with symptom improvement had a better outcome $(P=0.016)$. Pneumonia predicted a poor outcome $(P=0.021)$. Five patients expired and 22 patients suffered from recurrent stroke in the following 24 months. Previous transient ischemic attack (TIA) and anemia were associated with a higher risk of death or recurrent stroke within two years $(P=0.036$, $P=0.012$ ). Conclusion: High age, TACS, stroke in evolution and pneumonia were predictors for poor functional outcome. Previous TIA and anemia were predictors for death and recurrent stroke within two years.
\end{abstract}

Key words: Clinical outcome, internal carotid artery occlusion, recurrent stroke

Internal carotid artery (ICA) occlusion accounts for $6-15 \%$ of all acute cerebrovascular events and atherosclerosis is the most common cause. ${ }^{[1-3]}$ The clinical presentations of ICA occlusion are variable and include asymptomatic cases, transient ischemic attack (TIA), amaurosis fugax, minor or major disabling stroke and death. The efficiency of the collateral pathways is an important factor determining clinical presentation of ICA occlusion. ${ }^{[4,5]}$ Although several studies have discussed clinical presentation, vascular findings and collateral circulation in patients with ICA occlusion, only a few studies have been done to evaluate clinical outcome in acute stroke stage and the predictors for recurrent stroke. Paciaroni et al., reported that the presence of ipsilateral TIA, hypertension or hyperlipidemia was associated with a favorable outcome, ${ }^{[1]}$ but this study included all etiologies of ICA occlusion. However, different etiologies of ICA occlusion may be associated with different clinical outcomes. Due to atherosclerosis being the most common cause of ICA occlusion $(78 \%),{ }^{[1-3]}$ it is necessary to study the predictors of clinical outcome in atherosclerosis-related ICA occlusion, which is therefore not biased by other etiologies of ICA occlusion. To our knowledge, no studies have been performed on the predictors of clinical outcome in acute stroke stage and rate of recurrence specifically in atherosclerosisrelated ICA occlusion.

In this prospective study, we aimed (1) to assess the predictors of clinical functional outcome in patients of first-ever ischemic stroke with unilateral atherosclerosis-related ICA occlusion in acute stroke stage, (2) to evaluate the predictors of death and stroke recurrence within two years in these patients.

\section{Materials and Methods}

Between January 2002 and December 2003, patients with first-ever ischemic stroke associated with unilateral ICA occlusion were included prospectively from consecutive patients referred to the neurology department of the Keelung Chang Gung Memorial 
Hospital. Complete ICA occlusion was diagnosed when there was absence of flow in the ICA at the level of carotid bifurcation and detectable Doppler signal. All were confirmed by magnetic resonance (MR) angiography, computed tomography (CT) scan angiography or conventional cerebral angiography. ${ }^{[6-8]}$ The degree of contralateral ICA, contralateral common carotid artery (CCA) and ipsilateral CCA were calculated with ultrasound according to previous published criteria. ${ }^{[9]}$ In the acute phase, every patient underwent a CT scan of brain and received a complete neurological examination from an experienced neurologist. Brain CT or MR imaging was followed three to seven days after stroke onset. The diagnosis of ischemic stroke was made when the CT scan either showed a hypodense area corresponding to the clinical symptoms or was normal, whereas the neurological deficit was indicative of stroke. All patients had neurological deficit that lasted more than $24 \mathrm{~h}$. The recorded baseline characteristics included age, gender, hypertension (blood pressure over 140/90 mmHg at least twice before stroke or already under treatment with antihypertensive agent), diabetes mellitus (glucose levels $>140 \mathrm{mg} / \mathrm{dL}$ preprandial on two examinations, glucose level $>200 \mathrm{mg} / \mathrm{dl}$ postprandial or HbA1C $>8.5 \%$ ), cigarette smoking (current smoker or cessation less than five years before), previous ipsilateral TIA, coronary artery disease (history of acute myocardial infarction or angina pectoris), atrial fibrillation (presented on a standard 12-lead ECG), valvular heart disease (confirmed by echocardiography), anemia (hemoglobin levels $<13 \mathrm{~g} / \mathrm{dL}$ for men or $<12 \mathrm{~g} /$ $\mathrm{dL}$ for women), chronic renal insufficiency (creatinine $\geq 1.5 \mathrm{mg} / \mathrm{dL}$ ) and dyslipidemia (total cholesterol $\geq 200 \mathrm{mg} / \mathrm{dL}$ or $\geq 250 \mathrm{mg} / \mathrm{dL}$, low-density lipoprotein (LDL) $>130 \mathrm{mg} / \mathrm{dL}$, high-density lipoprotein (HDL) $<40 \mathrm{mg} / \mathrm{dL}$ ).

Cardioembolic stroke was diagnosed in patients with cardiac disorders associated with a significant risk of cerebral embolism, particularly atrial fibrillation, recent myocardial infarction and its thrombotic or cardiac wall sequelae, symptomatic valvular heart lesions or cardiomyopathy identified by echocardiography and the performance of cardiac interventional procedures. ${ }^{[10]}$ The diagnosis of carotid dissection was first suspected by duplex ultrasound, then confirmed by CT angiography or conventional angiography. ${ }^{[11-13]}$ Ischemic strokes not fulfilling the criteria for cardioembolism or strokes not attributable to other specific causes were categorized as caused by atherosclerosis.

The infarct territories involved were assessed by cerebral CT or MR images according to the criteria described by Tatu et al. ${ }^{[14]}$ Watershed infarcts were diagnosed according to published guidelines. ${ }^{[15]}$ At admission, the clinical subtypes of ischemic stroke were rated according to the classification of the Oxfordshire Community Stroke Project as partial anterior circulation syndrome (PACS), total anterior circulation syndrome (TACS), posterior circulation syndrome (POCS) and lacunar syndrome (LACS). ${ }^{[16]}$ Transient ischemic attack was defined as acute neurological deficit of vascular origin lasting less than $24 \mathrm{~h}$. Complications that happened during acute stroke stage were recorded as pneumonia (defined as fever, cough and consolidation or patchy interstitial pattern on chest X-ray), urinary tract infection (defined as dysuria accompanied by pyuria and bacteriuria), upper gastrointestinal bleeding (defined as a bloody nasogastric aspirate or tarry stool).

Patients that fulfilled the criteria of atherosclerosisrelated ICA occlusion were included. Cardioembolism, carotid dissection and other specific etiologies of ICA occlusion were excluded from this study. Patients were followed up prospectively at regular intervals for 24 months. End points of this study included recurrent ischemic stroke or death. Clinical functional outcome within 30 days from onset of first-ever ischemic stroke was assessed according to the modified Rankin Scale (mRS). ${ }^{[17]}$ "Functionally dependent" was defined as having an mRS score of 3, 4 or 5. All patients were regularly followed for at least two years. Follow-up consisted of clinical examinations at one and three months after stroke onset and then every three months. In case of a recurrent stroke, a clinical examination and cerebral CT scan were performed. Recurrent stroke was defined as any new focal neurological deficit of sudden onset lasting at least $24 \mathrm{~h}$ for which no other cause could be found other than ischemic stroke. A diagnosis of recurrence was not done where symptoms could be attributed to edema, mass effect, brain shift syndrome or hemorrhagic transformation and could not be diagnosed within $24 \mathrm{~h}$ of the index stroke. The causes of death were recorded.

\section{Statistical analysis}

Continuous variables were expressed as means \pm standard deviation. The associations between functional outcome within 30 days from stroke onset and categorical baseline variables, which included each stroke risk factor, infarct territories, clinical syndromes, acute complication and stroke symptoms, were calculated by Chi-square or Fisher's exact test. A $\mathrm{P}<0.05$ was considered statistically significant and was determined as predictor of functional outcome within 30 days from stroke onset. The crude associations between death and recurrent strokes within two years from stroke onset and each stroke risk factor, the degree of stenosis in other cerebral vessels were calculated by preliminary cross-tabulations with the Chi-square or Fisher's exact test. Multivariate logistic regression analysis was used to identify the independent predictors of death and stroke recurrence within two years from stroke onset and it was performed using the stepwise discriminant functional program: all variables with a $\mathrm{P}<0.05$ from 
the univariate analysis were retained. A value of $\mathrm{P}<0.05$ was considered statistically significant. All analyses were conducted using the SPSS/PC-win package.

\section{Results}

A total of 77 consecutive patients who had a firstever-ischemic stroke associated with unilateral ICA occlusion were included in the period January 2002 to December 2003. The presumed etiologies of ICA occlusion included atherosclerosis for 66 (85.7\%), cardioembolism for $10(12.9 \%)$ and artery dissection for one (1.3\%) patient. In terms of infarction territories, $15(19.5 \%)$ patients had entire middle cerebral artery (MCA) territory, eight (10.4\%) watershed, 12 (15.6\%) anterior superficial MCA territory, four (5.2\%) posterior superficial MCA territory, 11 (14.3\%) anterior and posterior MCA territory, four $(5.2 \%)$ striatocapsular, $19(24.6 \%)$ subcortical $(<1.5 \mathrm{~cm})$, one $(1.3 \%)$ anterior cerebral artery territory, two (2.6\%) brainstem and one $(1.3 \%)$ cerebellar infarction. For clinical stroke syndromes, $15(19.5 \%)$ patients presented with TACS, 34 (44.1\%) with PACS, 26 (33.8\%) with LACS, two (2.6\%) with POCS.

Among 77 first-ever ischemic stroke patients with unilateral ICA occlusion, 66 (85.7\%) patients whose etiology of ICA occlusion was presumed to be atherosclerosis were included in the subsequent analysis (baseline characteristics of atherosclerotic patients are summarized in Table 1). No patient died within 30 days after stroke onset. For 36 (54.5\%) patients, mRS score was 3 or higher within 30 days after stroke onset and the mean duration of hospital stay was $14.2 \pm 7.5$ (7-43) days. For 30 (45.5\%) patients, mRS score was lower than 3 and the mean duration of hospital stay was $8.7 \pm 5.8(0-16)$ days. Statistical analysis showed that higher age ( $\geq 70$ years) predicted a worse functional outcome $(P=0.049)$. Other risk factors of stroke, which included hypertension $(P=0.618)$, diabetes mellitus $(P=0.232)$, smoking $(P=0.349)$, ipsilateral TIA $(P=0.603)$, coronary artery disease $(P=0.272)$, anemia $(P=0.269)$, chronic renal insufficiency $(P=0.089)$ and dyslipidemia $(P=0.303)$ did not seem to influence clinical functional outcome. Of infarct territories, entire MCA territory infarction was associated with a poor functional outcome $(\mathrm{P}<0.001)$ within 30 days after stroke onset, while subcortical infarction $(<1.5 \mathrm{~cm})$ was associated with a better functional outcome $(P=0.016)$. Of clinical stroke syndromes, TACS was associated with a poor functional outcome $(P<0.001)$, but LACS was associated with a better functional outcome $(P=0.001)$. In acute stroke stage, stroke in evolution was associated with a poor functional outcome $(P=0.001)$, while stroke with symptoms' improvement was associated with a better functional outcome $(P=0.016)$. If pneumonia

\begin{tabular}{|c|c|}
\hline \multicolumn{2}{|c|}{$\begin{array}{l}\text { Table 1: Baseline characteristics of patients with } \\
\text { first-ever ischemic stroke associated with unilateral } \\
\text { atherosclerosis-related ICA occlusion }(n=66)\end{array}$} \\
\hline Parameter & $\mathrm{n}(\%)$ \\
\hline Mean age & $67.6 \pm 11.0$ \\
\hline Male & $51(77.3)$ \\
\hline \multicolumn{2}{|l|}{ Risk factors } \\
\hline Hypertension & $57(86.4)$ \\
\hline Diabetes mellitus & $18(27.3)$ \\
\hline Smoking & $38(57.6)$ \\
\hline Previous ipsilateral transient ischemic attack & $7(10.6)$ \\
\hline Coronary artery disease & $12(18.2)$ \\
\hline Anemia & $19(28.8)$ \\
\hline Chronic renal insufficiency & $10(15.2)$ \\
\hline \multicolumn{2}{|l|}{ Dyslipidemia (mg/dL) } \\
\hline Total cholesterol $\geq 200$ & $32(48.5)$ \\
\hline Total cholesterol $\geq 250$ & $8(12.1)$ \\
\hline Low-density lipoprotein >130 & $39(59.1)$ \\
\hline High-density lipoprotein $<40$ & $58(87.9)$ \\
\hline \multicolumn{2}{|l|}{ Infarct territories } \\
\hline Entire MCA territory & $12(18.2)$ \\
\hline Watershed infarcts & $7(10.6)$ \\
\hline Anterior superficial MCA & $10(15.2)$ \\
\hline Posterior superficial MCA & $4(6.1)$ \\
\hline Anterior + posterior MCA & $8(12.1)$ \\
\hline Striatocapsular & $4(6.1)$ \\
\hline Subcortical $(<1.5 \mathrm{~cm})$ & $17(25.8)$ \\
\hline Anterior cerebral artery & $1(1.5)$ \\
\hline Brainstem & $2(3.0)$ \\
\hline Cerebellum & $1(1.5)$ \\
\hline \multicolumn{2}{|l|}{ Clinical syndromes } \\
\hline Total anterior circulation syndrome & $12(18.2)$ \\
\hline Partial anterior circulation syndrome & $29(43.9)$ \\
\hline Lacunar syndromes & $23(34.8)$ \\
\hline Posterior circulation syndrome & $2(3.0)$ \\
\hline
\end{tabular}

MCA - middle cerebral artery; Data were given as mean \pm standard deviation or $\mathrm{n}(\%)$

happened in acute stroke stage, a poor functional outcome within 30 days after stroke onset could be predicted $(P=0.021)$. Peptic ulcer and urinary tract infection had no influence on functional outcome. Predictors of functional outcome within 30 days from stroke onset are summarized in Table 2.

Among the 66 first-ever ischemic stroke patients with unilateral atherosclerosis-related ICA occlusion, five (7.6\%) patients expired (range from Day 48 to 630) and $22(33.3 \%)$ patients suffered from nonfatal recurrent ischemic stroke in the following 24 months (range from Day 36 to 710 ). At the end of follow-up, nine (23.1\%) patients in the non-recurrent stroke group and $11(50 \%)$ patients in the recurrent stroke group were bedridden (mRS score 5). The causes of death were recurrent ischemic or hemorrhagic stroke (three), myocardial infarction (one) and septic shock (one). Multivariate analysis showed that previous ipsilateral TIA and anemia were associated with a higher risk of death or recurrent stroke within two years $(P=0.036$ and $P=0.012$, respectively). The degree of stenosis in contralateral ICA [Table 3], contralateral CCA and ipsilateral CCA (data not shown) had no influence on the risk of death or recurrent stroke in this analysis. Old age, male sex, hypertension, diabetes mellitus, smoking, 


\begin{tabular}{|c|c|c|c|c|}
\hline & mRS score $\geq 3$ ( $n=36)$ & mRS score $<3(n=30)$ & OR $(95 \% \mathrm{Cl})$ & $P$ value \\
\hline Mean age & $68.0 \pm 11.2$ & $67.1 \pm 10.9$ & $0.9(-4.58-6.38)$ & 0.747 \\
\hline Duration of acute hospital stay (days) & $14.2 \pm 7.5$ & $8.7 \pm 5.8$ & & \\
\hline Age $\geq 70$ & $24(66.7)$ & $13(43.3)$ & $2.62(0.96-7.12)$ & $0.049^{*}$ \\
\hline Males & $27(75.0)$ & $24(80.0)$ & $0.75(0.23-2.42)$ & 0.431 \\
\hline \multicolumn{5}{|l|}{ Infarct territories } \\
\hline Entire MCA territory & $12(40.0)$ & $0(0.0)$ & $0.44(0.33-0.59)$ & $<0.001^{*}$ \\
\hline Watershed infarcts & $3(8.3)$ & 4 (13.3) & $0.59(0.12-2.87)$ & 0.397 \\
\hline Anterior superficial MCA & $4(11.1)$ & $6(20.0)$ & $0.50(0.12-1.97)$ & 0.255 \\
\hline Posterior superficial MCA & $1(2.8)$ & $3(10.0)$ & $0.26(0.02-2.61)$ & 0.241 \\
\hline Anterior + posterior MCA & $6(16.7)$ & $2(6.7)$ & $2.80(0.52-15.04)$ & 0.196 \\
\hline Striatocapsular & $3(8.3)$ & $1(3.3)$ & $2.63(0.26-26.7)$ & 0.379 \\
\hline Subcortical $(<1.5 \mathrm{~cm})$ & $5(13.9)$ & $12(40)$ & $0.24(0.07-0.79)$ & $0.016^{*}$ \\
\hline \multicolumn{5}{|l|}{ Clinical syndromes } \\
\hline TACS & $12(33.3)$ & $0(0)$ & $0.44(0.33-0.59)$ & $<0.001^{*}$ \\
\hline PACS & $18(50)$ & $11(3.7)$ & $1.72(0.64-4.64)$ & 0.201 \\
\hline LACS & $6(16.7)$ & $17(56.7)$ & $0.15(0.04-0.47)$ & $0.001^{*}$ \\
\hline POCS & $0(0)$ & $2(6.7)$ & $0.43(0.33-0.57)$ & 0.203 \\
\hline \multicolumn{5}{|l|}{ Acute complications } \\
\hline Pneumonia & $6(16.7)$ & $0(0.0)$ & $0.50(0.38-0.64)$ & $0.021^{*}$ \\
\hline Peptic ulcer & 7 (19.4) & $3(10.0)$ & $2.17(0.50-9.26)$ & 0.238 \\
\hline Urinary tract infection & $1(2.8)$ & $1(3.3)$ & $0.82(0.05-13.83)$ & 0.706 \\
\hline \multicolumn{5}{|l|}{ Symptoms } \\
\hline In evolution & $16(44.4)$ & $2(6.7)$ & $11.2(2.31-54.26)$ & $0.001^{*}$ \\
\hline Improving & $0(0)$ & $5(16.7)$ & $0.41(0.30-0.55)$ & $0.016^{*}$ \\
\hline Stationary & $20(55.5)$ & $23(76.7)$ & $0.38(0.13-1.11)$ & 0.062 \\
\hline
\end{tabular}

mRS - modified Rankin scale; OR - odds ratio; $\mathrm{Cl}$ - confidence intervals; ${ }^{*} P<0.05$, Chi-square or Fisher's exact test; Data were given as mean \pm standard deviation or $\mathrm{n}(\%)$

\begin{tabular}{|c|c|c|c|c|}
\hline & $\begin{array}{l}\text { Death and stroke recurrence } \\
\qquad(\mathrm{n}=27)\end{array}$ & $\begin{array}{l}\text { No recurrent stroke } \\
\qquad(\mathrm{n}=39)\end{array}$ & OR $(95 \% \mathrm{CI})$ & $P$ value \\
\hline Mean age & $66.74 \pm 10.70$ & $68.18 \pm 10.34$ & $2.78(-6.9-4.1)$ & 0.892 \\
\hline Age $\geq 70$ & $14(51.8)$ & $23(58.9)$ & $0.75(0.28-2.01)$ & 0.374 \\
\hline Male & $21(77.8)$ & $30(76.9)$ & $1.05(0.32-3.39)$ & 0.589 \\
\hline \multicolumn{5}{|l|}{ Risk factors } \\
\hline Hypertension & $24(88.9)$ & $33(84.6)$ & $1.45(0.33-6.40)$ & 0.454 \\
\hline Diabetes mellitus & $8(29.6)$ & $10(25.6)$ & $1.22(0.41-3.65)$ & 0.466 \\
\hline Smoking & $16(59.2)$ & $22(56.4)$ & $1.12(0.42-3.04)$ & 0.510 \\
\hline Ipsilateral TIA & $6(22.2)$ & $1(2.5)$ & $10.85(1.22-96.33)$ & $0.016^{*}$ \\
\hline CAD & $5(18.5)$ & $7(17.9)$ & $1.03(0.29-3.69)$ & 0.599 \\
\hline Anemia & $12(44.4)$ & 7 (17.9) & $3.65(1.19-11.1)$ & $0.020^{*}$ \\
\hline CRI & $2(7.4)$ & $8(20.5)$ & $0.31(0.06-1.59)$ & 0.132 \\
\hline Total cholesterol $>200 \mathrm{mg} / \mathrm{dL}$ & $12(30.7)$ & $20(74.1)$ & $0.76(0.28-2.03)$ & 0.384 \\
\hline \multicolumn{5}{|l|}{ Contralateral ICA } \\
\hline No stenosis & $22(81.5)$ & $22(56.4)$ & $3.40(1.06-10.83)$ & $0.030^{*}$ \\
\hline$<50 \%$ stenosis & $2(7.4)$ & $13(33.3)$ & $0.16(0.03-0.78)$ & $0.012^{*}$ \\
\hline $50-69 \%$ stenosis & $1(3.7)$ & $1(2.6)$ & $1.46(0.08-24.43)$ & 0.655 \\
\hline $70-99 \%$ stenosis & $2(7.4)$ & $3(7.7)$ & $0.96(0.14-6.17)$ & 0.672 \\
\hline \multicolumn{5}{|l|}{ Multivariate logistic regression } \\
\hline Ipsilateral TIA & & & $11.10(1.17-104.63)$ & $0.036^{\dagger}$ \\
\hline Anemia & & & $5.09(1.43-18.07)$ & $0.012^{\dagger}$ \\
\hline
\end{tabular}

ICA - internal carotid artery; CCA - common carotid artery, ${ }^{\star} P<0.05$, Chi-square or Fisher's exact test; ${ }^{\dagger} P<0.05$, multivariate logistic regression; Data were given as mean \pm standard deviation or $\mathrm{n}(\%)$

coronary artery disease, chronic renal insufficiency or hyperlipidemia was not associated with a higher rate of death or recurrent stroke within two years after stroke onset [Table 3].

\section{Discussion}

This prospective stroke study showed that age older than 70 years, entire MCA territory infarction, TACS, stroke in evolution and pneumonia are associated with dependent outcome within one month after stroke onset in patients with unilateral atherosclerosis-related ICA occlusion. In addition, subcortical infarction, LACS and initial symptoms with improvement can predict an independent outcome. Furthermore, previous ipsilateral TIA and anemia are associated with higher death or recurrent stroke rate within two years.

There are numerous previous stroke studies on 
the prognostic factors for clinical outcome. Old age, stroke severity, heart diseases and dementia have an impact on mortality and stroke recurrence. ${ }^{[18]}$ Only few studies were done specifically in patients with ICA occlusion. Previous reports found that patients with ICA occlusion had a variable clinical course and higher rate of recurrent stroke. ${ }^{[19]}$ Hypertension might hinder the development of preformed intracranial collateral vessels. ${ }^{[20]}$ Hypercholesterolemia and previous TIA in the same vascular territory have been associated with an improved functional outcome but was controversy. ${ }^{[21,22]}$ The onset of ICA occlusion might correlate with a potentially fatal outcome; once the occlusion has been established, the subsequent risk of stroke seems lower. ${ }^{[23]}$ Age can predict mortality within 30 days after stroke onset in patients with ICA occlusion. ${ }^{[1]}$ In addition, previous ipsilateral TIA, younger age and dyslipidemia were associated with a favorable functional outcome. ${ }^{[1]}$ However, different etiologies of ICA occlusion may influence clinical outcome and no previous study was performed specifically on atherosclerosis-related ICA occlusion. Our study was performed specifically on patients of first-ever ischemic stroke with unilateral atherosclerosis-related ICA occlusion. We found that age older than 70 years can predict dependent functional outcome within one month after stroke onset, a finding similar to previous studies. ${ }^{[1,18]}$ We also suggested that the size of cerebral infarction and the severity of clinical stroke syndrome affect clinical functional outcome. If the infarct size is large, as in the entire MCA territory infarction, the outcome is poor. If the initial presentation is severe, as in TACS, the outcome is dependent.

Pneumonia, which is a common complication in acute stroke stage, was associated with a poor outcome within one month after stroke onset in our study. This finding may be due to aspiration pneumonia, which happens more frequently when the stroke severity is high and the degree of dysphagia is severe. Another possible explanation is that severe pneumonia might cause hypoxemia, which aggravates cerebral hypoxia and leads to cerebral morbidity. Intensive chest care and prevention of aspiration pneumonia in acute stroke stage may improve clinical outcome.

The risk of recurrent stroke in patients with ICA occlusion was about $7.0 \%$ per year and the risk of stroke ipsilateral to the carotid occlusion was $5.9 \% .^{[15,24-27]}$ Previous TIA and presence of leptomeningeal collaterals may predict future cerebral ischemia. ${ }^{[28-30]}$ The risk of stroke increases with blood pressure in the great majority of patients with symptomatic carotid artery disease. ${ }^{[31]}$ However, the relationship between blood pressure and stroke risk was inverted in patients with bilateral carotid stenosis by more than $70 \% .{ }^{[2-34]}$ The risk of recurrent stroke is high in ICA occlusion patients who have high volume flow to the brain and increased collateral circulations. ${ }^{[35]}$ Watershed infarcts might be predictors of stroke recurrence. ${ }^{[15,36]}$ In our study, previous ipsilateral TIA could predict recurrent stroke in patients with unilateral ICA occlusion, a finding similar to previous studies. ${ }^{[1,29]}$ Anemia is associated with high risk of stroke recurrence. We have no good explanation for this finding since we did not classify the etiologies of anemia in detail, but bleeding with subsequent anemia might be a risk factor for cerebral infarction. ${ }^{[37]}$ As in the previous studies, ${ }^{[1,19,23]}$ the degree of stenosis in other carotid vessels did not influence stroke recurrence.

Our study has some limitations. First, the data are from a single hospital registry. As usually seen in hospitalbased stroke registries, our series is probably biased in some categories of stroke. Another drawback of the study is the relatively small number of patients and this could potentially have influenced the statistical analysis.

\section{Conclusion}

In summary, age older than 70 years, infarct territory as entire MCA territory infarction, clinical stroke syndrome as TACS, initial stroke symptoms in evolution and pneumonia in acute stroke stage are associated with dependent clinical functional outcome within one month after the onset of first-ever ischemic stroke in patients with unilateral atherosclerosis-related ICA occlusion. Previous ipsilateral TIA and anemia can predict stroke recurrence within 3 years following firstever ischemic stroke in these patients.

\section{Acknowledgments}

We thank all the collaborators in this original study. We also thank the research nurses for help with the collection of the data.

\section{References}

1. Paciaroni M, Caso V, Venti M, Milia P, Kappelle LJ, Silvestrelli G, \& al. Outcome in patients with stroke associated with internal carotid artery occlusion. Cerebrovasc Dis 2005;20:108-13.

2. Pessin MS, Duncan GW, Mohr JP, Poskanzer DC. Clinical and angiographic features of carotid transient ischemic attacks. N Engl J Med 1977;296:358-62.

3. Mead GE, Murray H, Farrell A, O’Neill PA, McCollum CN. Pilot study of carotid surgery for acute stroke. Br J Surg 1997;84:990-2.

4. Bisschops RH, Klijn CJ, Kappelle LJ, van Huffelen AC, van der Grond J. Collateral flow and ischemic brain lesions in patients with unilateral carotid artery occlusion. Neurology 2003;60:1435-41.

5. Powers W. Cerebral hemodynamies in ischemic cerebrovascular disease. Ann Neurol 1991;29:231-40.

6. Paciaroni M, Caso V, Cardaioli G, Corea F, Milia P, Venti M, \& al. Is ultrasound examination sufficient in the evaluation of patients with internal carotid artery severe stenosis or occlusion? Cerebrovasc Dis 2003;15:173-6.

7. Anderson GB, Ashforth R, Steinke DE, Ferdinandy R, Findlay JM. CT Angiography for the detection and characterization of carotid artery bifurcation disease. Stroke 2000;31:2168-74. 
8. Sameshima T, Futami S, Morita Y, Yokogami K, Miyahara S, Sameshima Y, \& al. Clinical usefulness of and problems with threedimensional CT angiography for the evaluation of arteriosclerotic stenosis of the carotid artery: Comparison with conventional angiography, MRA and ultrasound sonography. Surg Neurol 1999;51:301-8.

9. Eliasziw M, Rankin RN, Fox AJ, Haynes RB, Barnett HJ. Accuracy and prognostic consequences of ultrasonography in identifying severe carotid artery stenosis. Stroke 1995;26:1747-52.

10. Barnett HJ, Gunton RW, Eliasziw M, Fleming L, Sharpe B, Gates P, \& al. Causes and severity of ischemic stroke in patients with internal carotid artery stenosis. JAMA 2000;283:1429-36.

11. Kirsch E, Kaim A, Engelter S, Lyrer P, Stock KW, Bongartz G, \& al. MR angiography in internal carotid artery dissection: Improvement of diagnosis by selective demonstration of the intramural haematoma. Neuroradiology 1998;40:704-9.

12. Stringaris K, Liberopoulos K, Giaka E, Kokkinis K, Bastounis E, Klonaris EC, \& al. Three-dimensional time-of-flight MR angiography and MR imaging versus conventional angiography in carotid artery dissections. Int Angiol 1996;15:20-5.

13. Sturzenegger M, Mattle HP, Rivoir A, Baumgartner RW. Ultrasound findings in carotid artery dissection: Analysis of 43 patients. Neurology 1995;45:691-8

14. Tatu L, Moulin T, Bogousslavsky J, Duvernoy H. Arterial territories of the human brain: Cerebral hemispheres. Neurology 1998;50:1699-708.

15. Bogousslavsky J, Regli F. Borderzone infarctions distal to internal carotid artery occlusion: Prognostic implications. Ann Neurol 1986;20:346-50.

16. Bamford J, Sandercock P, Dennis M, Burn J, Warlow C. Classification and natural history of clinically identifiable subtypes of cerebral infarction. Lancet 1991;337:1521-6.

17. de Haan R, Limburg M, Bossuyt P, van der Meulen J, Aaronson N. The clinical meaning of Rankin ,handicap' grades after stroke. Stroke 1995;26:2027-30.

18. Appelros P, Nydevik I, Viitanen M. Poor outcome after first-ever stroke: Predictors for death, dependency and recurrent stroke within the first year. Stroke 2003;34:122-6.

19. Faught WE, van Bemmelen PS, Mattos MA, Hodgson KJ, Barkmeier LD, Ramsey DE, $\notin$ al. Presentation and natural history of internal carotid artery occlusion. J Vasc Surg 1993;18:512-23.

20. Hedera P, Bujdáková J, Traubner P, Pancák J. Stroke risk factors and development of collateral flow in carotid occlusive disease. Acta Neurol Scand 1998;98:182-6.

21. Vauthey C, de Freitas GR, van Melle G, Devuyst G, Bogousslavsky J. Better outcome after stroke with higher serum cholesterol levels. Neurology 2000;54:1944-9.

22. Weih M, Kallenberg K, Bergk A, Dirnagl U, Harms L, Wernecke KD, e al. Attenuated stroke severity after prodromal TIA: A role for ischemic tolerance in the brain? Stroke 1999;30:1851-4.

23. Verlato F, Camporese G, Bernardi E, Salmistraro G, Roceo S, Mayellaro V, \& al. Clinical outcome of patients with internal carotid artery occlusion:
A prospective follow-up study. J Vase Surg 2000;32:293-8.

24. Fields WS, Lemak NA. Joint study of extracranial arterial occlusion, X: Internal carotid artery occlusion. JAMA 1976;235:2734-8.

25. Milandre L, Pestre P, Botti G, Alichérif A, Khalil R. Carotid artery occlusion disclosed by cerebral ischemia. Lesional, etiologic and developing aspects. Ann Med Intern 1990;141:115-22.

26. Cote R, Barnett HJ, Taylor DW. Internal carotid occlusion: A prospective study. Stroke 1983;14:898-902

27. Samson D, Watts C, Clark K. Cerebral revascularization for transient ischemic attacks. Neurology 1977;27:767-71.

28. Yamauchi H, Fukuyama H, Nagahama Y, Nabatame H, Ueno M, Nishizawa S, \& al. Significance of increased oxygen extraction fraction in five-year prognosis of major cerebral arterial occlusive diseases. J Nucl Med 1999;40:1992-8.

29. Klijn CJ, Kappelle LJ, van Huffelen AC, Visser GH, Algra A, Tulleken CA, \&al. Recurrent ischemia in symptomatic carotid occlusion: Prognostic value of hemodynamic factors. Neurology 2000;55:1806-12.

30. Grubb RL Jr, Derdeyn CP, Fritsch SM, Carpenter DA, Yundt KD, Videen TO, $\mathbb{A}$ al. Importance of hemodynamic factors in the prognosis of symptomatic carotid occlusion. JAMA 1998;280:1055-60.

31. Rothwell PM, Howard SC, Spence JD; Carotid Endarterectomy trialists' Collaboration. Relationship between blood pressure and stroke risk in patients with symptomatic carotid occlusive disease. Stroke $2003 ; 34: 2583-90$

32. Franklin SS, Sutton-Tyrrell K, Belle SH, Weber MA, Kuller LH. The importance of pulsatile components of hypertension in predicting carotid stenosis in older adults. J Hypertens 1997;15:1143-50.

33. van Popele NM, Grobbee DE, Bots ML, Asmar R, Topouchian J, Reneman RS, etal. Association between arterial stiffness and atherosclerosis: The Rotterdam Study. Stroke 2001;32:454-60.

34. Safar ME, Blacher J, Mourad JJ, London GM. Stiffness of carotid artery wall material and blood pressure in humans: Application to antihypertensive therapy and stroke prevention. Stroke 2000;31:78290

35. Rutgers DR, Klijn CJ, Kappelle LJ, van der Grond J. Recurrent stroke in patients with symptomatic carotid artery occlusion is associated with high-volume flow to the brain and increased collateral circulation. Stroke 2004;35:1345-9.

36. Klijn CJ, Kappelle LJ, van der Grond J, Algra A, Tulleken CA, van Gijn J. Magnetic resonance techniques for the identification of patients with symptomatic carotid artery occlusion at high risk of cerebral ischemic events. Stroke 2000;31:3001-7.

37. Kim JS, Kang SY. Bleeding and subsequent anemia: A precipitant for cerebral infarction. Eur Neurol 2000;43:201-8.

Accepted on 31-01-2008

Source of Support: Nil, Conflict of Interest: None declared. 